\title{
Large zero-field spin splitting in AIGaN/AIN/GaN/AIN heterostructures
}

S. B. Lisesivdin, N. Balkan, O. Makarovsky, A. Patanè, A. Yildiz, M. D. Caliskan, M. Kasap, S. Ozcelik, and E. Ozbay

Citation: Journal of Applied Physics 105, 093701 (2009);

View online: https://doi.org/10.1063/1.3120782

View Table of Contents: http://aip.scitation.org/toc/jap/105/9

Published by the American Institute of Physics

\section{Articles you may be interested in}

Zero-field spin splitting in $\mathrm{Al}_{x} \mathrm{Ga}_{1-x} \mathrm{~N} / \mathrm{GaN}$ heterostructures with various $\mathrm{Al}$ compositions Applied Physics Letters 93, 172113 (2008); 10.1063/1.3012361

Weak antilocalization and beating pattern in high electron mobility $\mathrm{Al}_{x} \mathrm{Ga}_{1-x} \mathrm{~N} / \mathrm{GaN}$ two-dimensional electron gas with strong Rashba spin-orbit coupling

Journal of Applied Physics 104, 053703 (2008); 10.1063/1.2974091

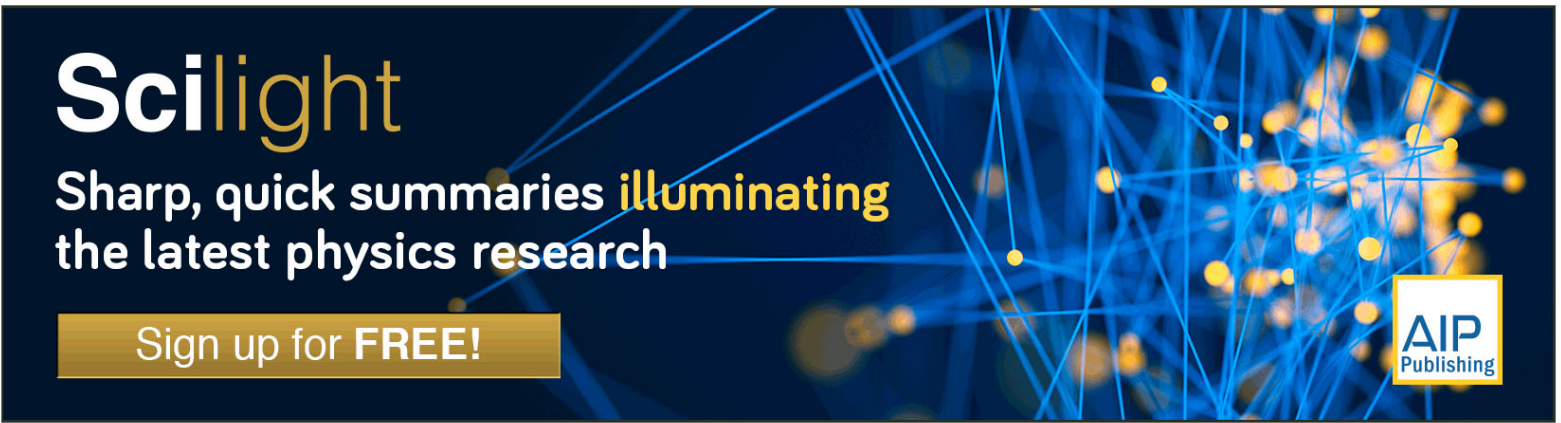




\title{
Large zero-field spin splitting in AIGaN/AIN/GaN/AIN heterostructures
}

\author{
S. B. Lisesivdin, ${ }^{1,2, a)}$ N. Balkan, ${ }^{1}$ O. Makarovsky, ${ }^{3}$ A. Patanè,${ }^{3}$ A. Yildiz, ${ }^{4}$ M. D. Caliskan, ${ }^{5}$ \\ M. Kasap, ${ }^{2}$ S. Ozcelik, ${ }^{2}$ and E. Ozbay,6 \\ ${ }^{1}$ Department of Computing and Electronic Systems, University of Essex, CO4 3SQ Colchester, United \\ Kingdom \\ ${ }^{2}$ Department of Physics, Gazi University, Teknikokullar, 06500 Ankara, Turkey \\ ${ }^{3}$ School of Physics and Astronomy, University of Nottingham, NG7 2RD, United Kingdom \\ ${ }^{4}$ Department of Physics, Faculty of Science and Arts, Ahi Evran University, Asikpasa Kampusu, 40040 \\ Kirsehir, Turkey \\ ${ }^{5}$ Nanotechnology Research Center, Bilkent University, Bilkent, 06800 Ankara, Turkey \\ ${ }^{6}$ Department of Physics, Department of Electrical and Electronics Engineering, Bilkent University, Bilkent, \\ 06800 Ankara, Turkey
}

(Received 30 October 2008; accepted 23 March 2009; published online 1 May 2009)

\begin{abstract}
This work describes Shubnikov-de Haas (SdH) measurements in $\mathrm{Al}_{0.22} \mathrm{Ga}_{0.78} \mathrm{~N} / \mathrm{AlN} / \mathrm{GaN} / \mathrm{AlN}$ heterostructures. Our experiments coupled with the analysis of the Hall data at various temperatures confirm the formation of a two-dimensional electron gas (2DEG) at the AlN/GaN interface. A beating pattern in the $\mathrm{SdH}$ oscillations is also observed and attributed to a zero-field spin splitting of the 2DEG first energy subband. The values of the effective spin-orbit coupling parameter and zero-field spin-split energy are estimated and compared with those reported in the literature. We show that zero-field spin-split energy tends to increase with increasing sheet electron density and that our value $(12.75 \mathrm{meV})$ is the largest one reported in the literature for GaN-based heterostructures. (C) 2009 American Institute of Physics. [DOI: 10.1063/1.3120782]
\end{abstract}

\section{INTRODUCTION}

The electronic properties of two-dimensional electron gases (2DEGs) in $\mathrm{Al}_{x} \mathrm{Ga}_{1-x} \mathrm{~N} / \mathrm{GaN}, \quad \mathrm{Al}_{x} \mathrm{Ga}_{1-x} \mathrm{~N} / \mathrm{AlN} /$ $\mathrm{GaN}$, and $\mathrm{Al}_{x} \mathrm{Ga}_{1-x} \mathrm{~N} / \mathrm{AlN} / \mathrm{GaN} / \mathrm{AlN}$ heterostructures have been studied extensively for applications in high-power, high-temperature microwave devices and high-frequency high electron mobility transistors (HEMTs). ${ }^{1-4}$ Recent improvements in transport parameters, such as mobility and sheet electron density, have also led to increased interest in studies of the quantum Hall effect. ${ }^{5,6}$ Due to the existence of high electrical fields caused by piezoelectric and spontaneous polarization, GaN-based heterostructures have an intrinsic 2DEG with high sheet carrier densities. ${ }^{7,8}$ These electric fields also enhance the spin-orbit interaction thus leading to a finite spin energy splitting of the conduction band states even in the absence of externally applied magnetic fields. ${ }^{9,10}$ Zero spin splitting plays a key role in the high Curie temperatures and ferromagnetic properties reported in previous studies of wide bandgap semiconductors, such as $\mathrm{GaN}$ and $\mathrm{ZnO} .^{11,12}$ Therefore GaN-based heterostructures are also promising candidates for applications in spintronics.

Spin-orbit interaction and spin splitting in zincblende III-V materials have been studied extensively and are understood to a high degree. ${ }^{13}$ Unlike the zincblende structures, in wurtzite low dimensional structures, there is an extra spin splitting term due to the lack of inversion symmetry in the wurtzite-type lattice. ${ }^{12}$ Recent experimental work indicate a serious disagreement between the results obtained from Shubnikov-de Haas $(\mathrm{SdH})^{14-18}$ and weak antilocalization

\footnotetext{
a) Author to whom correspondence should be addressed. Electronic mail: sblisesivdin@gmail.com.
}

(WAL) ${ }^{10,17,19-22}$ measurements. Also, circular photogalvanic ${ }^{23}$ measurements suggest that spin-splitting energy values should lie in the range from 0.1 to $9 \mathrm{meV}^{18,21}$ The reason for the disagreement and conflicting results is yet to be established.

The theoretical estimation for the Rashba dominated spin-splitting energy is, however, only $\sim 1 \mathrm{meV} .^{24}$ Lo et $\mathrm{al}^{25}$ proposed an additional spin-splitting mechanism called $\Delta_{C 1}-\Delta_{C 3}$ coupling, where the band folding generates two conduction bands for the wurtzite structures and the coupling between these conduction bands enhances the spin-splitting energy. Therefore, Lo et al. concluded that the band folding and lack of inversion symmetry significantly contribute to the spin splitting in wurtzite GaN-based quantum wells. ${ }^{25}$

In this work, we report a detailed study of $\mathrm{SdH}$ oscillations in an AlGaN/AlN/GaN/AlN heterostructure with high Hall mobility and sheet carrier density. Our experiment and analysis allow us to estimate the effective spin-orbit coupling parameter and zero-field spin-split energy, $E_{\uparrow}-E_{\downarrow}$. To our knowledge, our value of $E_{\uparrow}-E_{\downarrow} \sim 13 \mathrm{meV}$ is the largest value reported in the literature for GaN-based heterostructures. This result is relevant for future developments of GaNbased spintronic devices, such as spin-polarized field effect transistors.

\section{EXPERIMENTAL TECHNIQUES}

The samples used in the present study were all grown on c-plane (0001) sapphire $\left(\mathrm{Al}_{2} \mathrm{O}_{3}\right)$ substrate by low-pressure Metal-Organic Chemical Vapor Deposition (MOCVD). Hydrogen was used as the carrier gas and trimethylgallium, trimethylaluminum, and ammonia were used as $\mathrm{Ga}, \mathrm{Al}$, and $\mathrm{N}$ precursors, respectively. The substrate was cleaned in $\mathrm{H}_{2}$ am- 


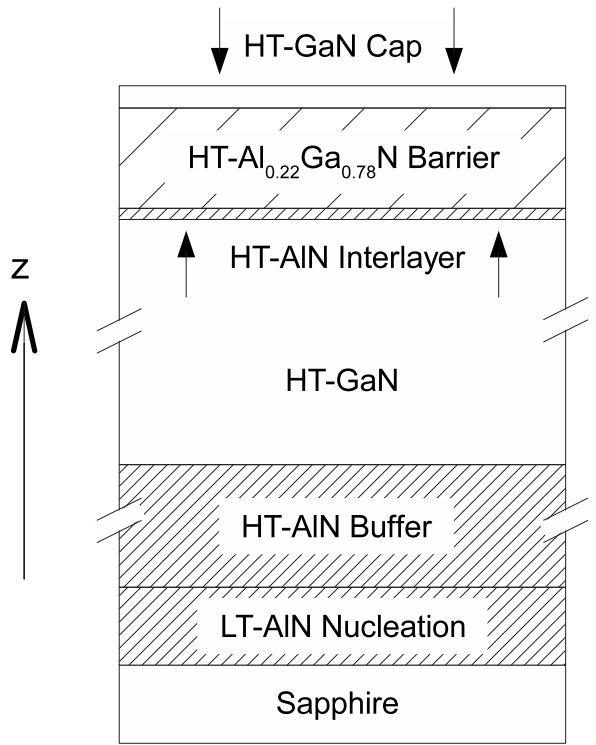

FIG. 1. Layer structure of our samples.

bient at $1100{ }^{\circ} \mathrm{C}$ and then a $15 \mathrm{~nm}$ low-temperature (LT) AlN nucleation layer was grown at $840{ }^{\circ} \mathrm{C}$ with $50 \mathrm{mbar}$ reactor pressure. After the deposition of the LT-AIN nucleation layer, the wafers were heated to a high temperature (HT) for annealing. For all samples, a HT-AlN buffer layer of thickness of about $0.60 \mu \mathrm{m}$ was deposited on the annealed nucleation layers at $1127{ }^{\circ} \mathrm{C}$ with a $400 \mathrm{~nm} / \mathrm{h}$ growth rate. After the deposition of the buffer layer, a HT-GaN layer with an approximate thickness of $1.9 \mu \mathrm{m}$ was grown at $1040{ }^{\circ} \mathrm{C}$. Finally, a $1.5 \mathrm{~nm}$ thick AlN interlayer, a $27 \mathrm{~nm}$ thick $\mathrm{Al}_{0.22} \mathrm{Ga}_{0.78} \mathrm{~N}$ barrier layer, and a $3 \mathrm{~nm} \mathrm{GaN}$ cap layer were grown at $1080{ }^{\circ} \mathrm{C}$. All layers were nominally undoped. Details of the sample layer structure are shown in Fig. 1.

For the resistivity and Hall measurements by the Van der Pauw method, square shaped $\left(5 \times 5 \mathrm{~mm}^{2}\right)$ samples were prepared with four evaporated and annealed Ti/Al/Ni/Au (200 $\AA / 2000 \AA / 300 \AA / 700 \AA$ ) Ohmic contacts in the corners. Gold wires were soldered to the contacts with indium. The Ohmic behavior of the contacts was confirmed by the form of the current voltage characteristics, which are symmetrical with respect to the polarity of the applied voltage. Hall measurements were carried out in the temperature range of $2-350 \mathrm{~K}$. The Hall coefficient and resistivity were measured for both directions of current, magnetic field polarizations, and for all possible contact configurations.

\section{RESULTS AND DISCUSSION}

Figure 2 shows the temperature dependence of the Hall mobility and Hall sheet carrier density for the samples investigated in the current study. LT (2 K) Hall mobility and Hall sheet carrier density are $19950 \mathrm{~cm}^{2} / \mathrm{V} \mathrm{s}$ and 1.05 $\times 10^{13} \mathrm{~cm}^{-2}$, respectively. As shown in Fig. 2, the Hall mobility is nearly temperature independent below $100 \mathrm{~K}$, but decreases with increasing temperature above $100 \mathrm{~K}$, as expected from an increasing dominance of polar optical phonon scattering. In addition, the sheet carrier density is nearly independent of temperature in the whole range of temperatures, except at HTs where it tends to increase slightly due to

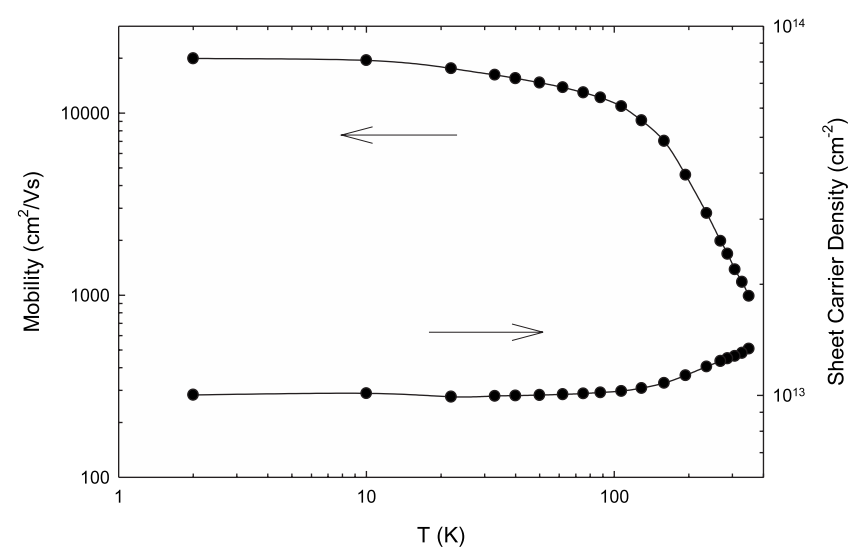

FIG. 2. Temperature dependent Hall mobility and Hall sheet carrier density of our samples. Lines are guides to the eyes.

thermal activation of bulk carriers. The behavior of both mobility and sheet carrier density is typical of 2DEGs.

Figure 3 shows a typical longitudinal magnetoresistivity measurement $\left[\rho_{x x}\left(B_{z}\right)\right]$ as a function of a perpendicular magnetic field, $B_{z}$, at $2 \mathrm{~K}$. The $\mathrm{SdH}$ oscillations at magnetic fields between 8 and $14 \mathrm{~T}$ are shown in the insert of Fig. 3. The negative parabolic magnetoresistance in this magnetic field range was also observed by Brana et al. ${ }^{26}$ and attributed to diffusive electron-electron interactions. In order to extract the contribution of the parabolic background magnetoresistance from the $\mathrm{SdH}$ oscillations, we used a general method and calculated the negative second derivative $\left(-\partial^{2} R_{x x} / \partial B^{2}\right)$ of the raw magnetic field dependent experimental data with respect to magnetic field and plotted the results as a function of inverse magnetic field in Fig. $4 .{ }^{27-30}$ The beating pattern superimposed on the periodic $\mathrm{SdH}$ oscillations is clearly seen in this figure. In order to obtain the characteristic frequencies of these oscillations, we used fast Fourier transform (FFT) analysis. The results of the FFT analysis are shown in the insert of Fig. 4. We obtain two distinct $\mathrm{SdH}$ frequencies $f_{1}$ $=202.7$ and $f_{2}=226.1 \mathrm{~T}$.

For structures similar to ours, Lo et al. ${ }^{15}$ proposed three possible mechanisms for the beating effect: (i) spin splitting of the 2DEG first energy subband, (ii) two-subbands occupa-

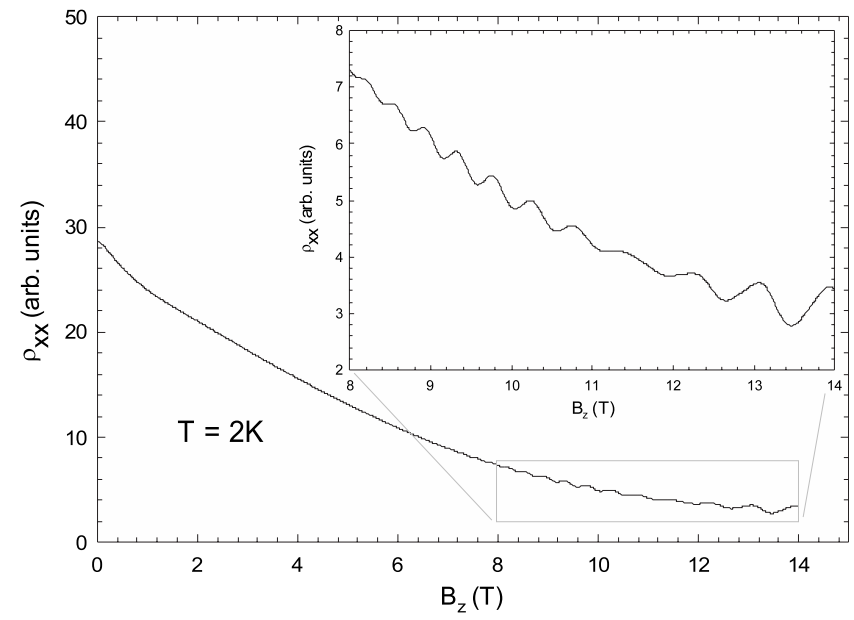

FIG. 3. Magnetic field dependent longitudinal magnetoresistivity $\left[\rho_{x x}\left(B_{z}\right)\right]$ at $2 \mathrm{~K}$. Insert: $\mathrm{SdH}$ oscillations in the range of magnetic fields $8-14 \mathrm{~T}$. 


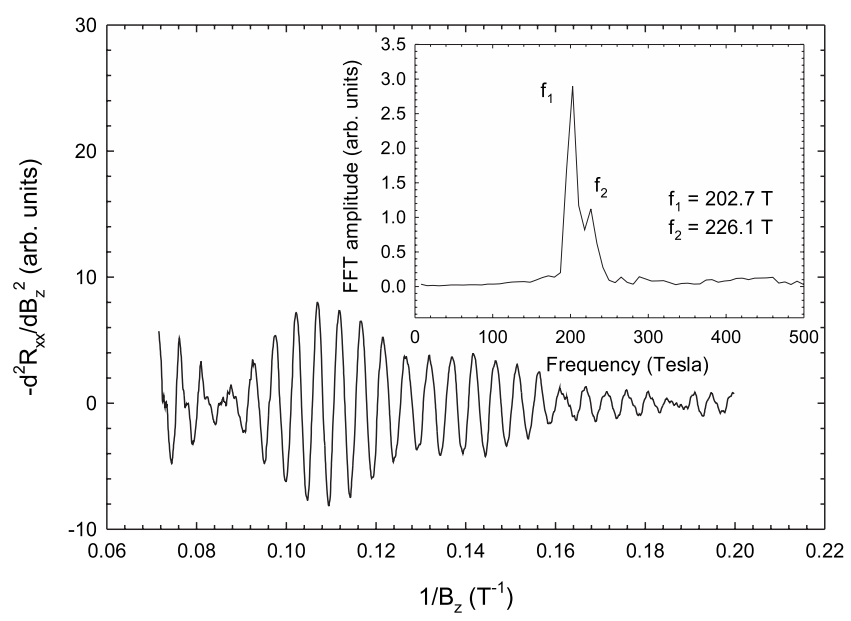

FIG. 4. The negative second derivative of the magnetoresistance $\left[R_{x x}\left(B_{z}\right)\right]$ with respect to an inverse magnetic field. The insert shows two main frequencies derived from the Fourier analysis of the the $\mathrm{SdH}$ oscillations.

tion, and (iii) second 2D channel located in the barrier layer. A fourth possible mechanism is the existence of a twodimensional hole gas (2DHG) between the GaN and AlN buffer layers. ${ }^{31}$ In order to establish the existence of this 2DHG, magnetic field dependent Hall measurements were carried out and the results were analyzed using the quantitative mobility spectrum analysis (QMSA) technique reported by us in our previous studies on similar GaN-based heterostructures. ${ }^{32-34}$ Mobility spectra at temperatures $T$ $=22 \mathrm{~K}$ and $T=159 \mathrm{~K}$ are shown in Figs. 5(a) and 5(b), respectively. It is clear from the figure that the $2 \mathrm{DEG}$ conduction is dominant and there is a negligible contribution from the hole channel. Therefore we infer that Hall mobility and Hall sheet carrier density shown in Fig. 2 are due only to the 2DEG at the AlN/GaN heterojunction. The existence of a 2D channel in the barrier layer is also dismissed through a lack of evidence in the QMSA analysis. The second potential source for the observed beating is the double subband occupancy, which we exclude for the following reasons. Using the well known relation

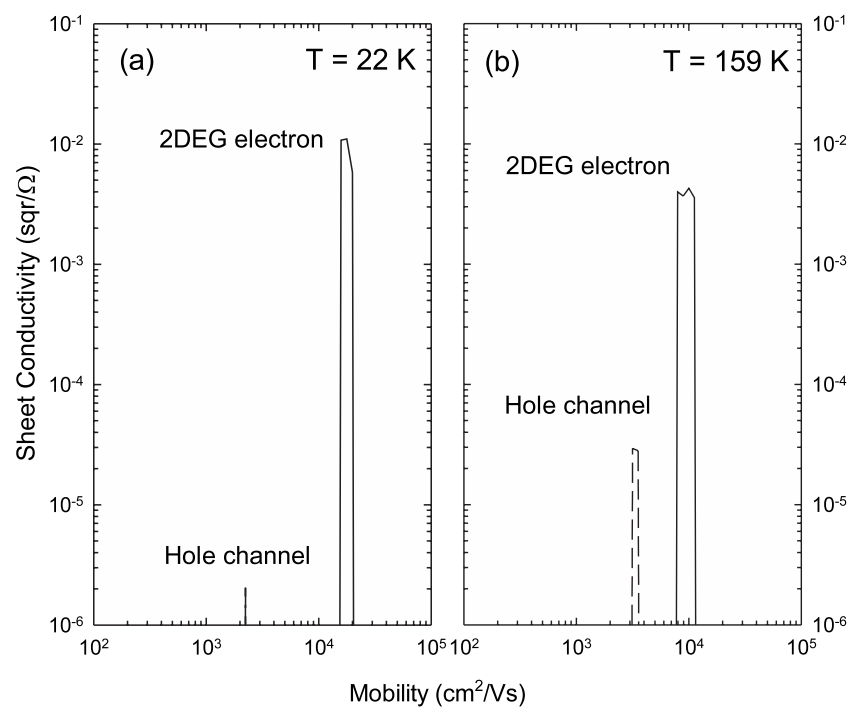

FIG. 5. Electron (solid lines) and hole (dashed lines) QMSA spectra at (a) 22 and (b) $159 \mathrm{~K}$.
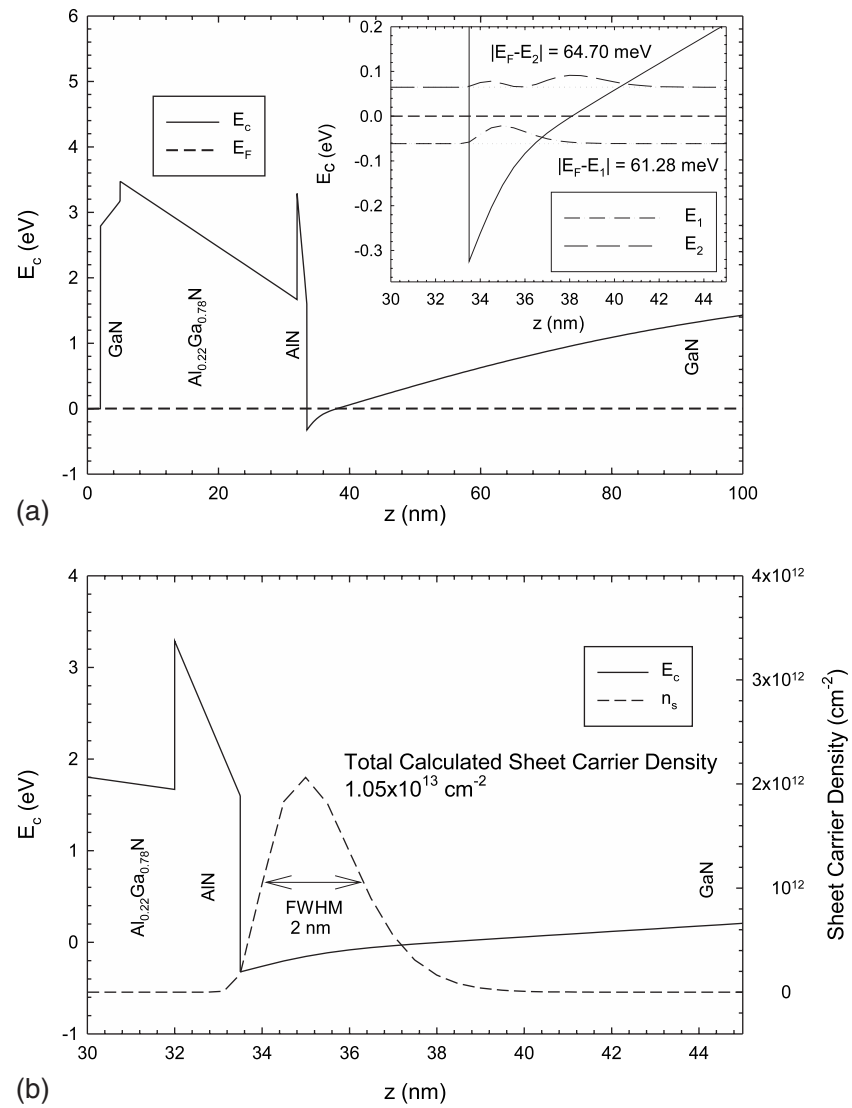

FIG. 6. (a) Conduction band profile (full line) and Fermi level (dashed line) for our $\mathrm{Al}_{0.22} \mathrm{Ga}_{0.78} \mathrm{~N} / \mathrm{AlN} / \mathrm{GaN} / \mathrm{AlN}$ heterostructure. Insert: first two subband energies and Fermi level and related electron wave functions. (b) Conduction band profile (full line) and calculated sheet carrier density (dashed line).

$$
\Delta_{i}\left(\frac{1}{B}\right)=\frac{e}{\pi \hbar n_{i}},
$$

we calculated the electron density for the two subbands by using the $\mathrm{SdH}$ frequencies shown in the insert of Fig. $4 .^{35}$ Here, $n_{i}$ is the carrier density of the $i$ th subband and $\Delta_{i}$ is the period in $1 / B$ of the SdH oscillations. Using Eq. (1), we find that the carrier densities of the first two energy subbands are $n_{1}=1.09 \times 10^{13}$ and $n_{2}=9.77 \times 10^{12} \mathrm{~cm}^{-2}$. The total carrier density is $n_{1}+n_{2} \cong 2.07 \times 10^{13} \mathrm{~cm}^{-2}$ and is much larger than the Hall sheet carrier density of $1.05 \times 10^{13} \mathrm{~cm}^{-2}$ shown in Fig. 2. Therefore, the beating effect cannot be related to the two-subband occupancy. In order to further justify this observation, we solved self-consistently the nonlinear Schrödinger-Poisson equations for our $\mathrm{Al}_{0.22} \mathrm{Ga}_{0.78} \mathrm{~N} /$ AlN/GaN/AlN HEMT structure. ${ }^{36}$ The results are shown in Fig. 6. The subband energies and related electron wave functions are shown in the insert of Fig. 6(a) for $T=2 \mathrm{~K}$. As shown in Fig. 6(a), the Fermi level is $64.70 \mathrm{meV}$ below the second subband. The first subband is $61.28 \mathrm{meV}$ below the Fermi level and is occupied at $2 \mathrm{~K}$. The energy separation between the two subbands is $E_{2}-E_{1}=125.98 \mathrm{meV}$. This value is similar to both theoretical and experimental results reported for the $\mathrm{AlGaN} / \mathrm{GaN}$ structures by several groups. ${ }^{15,37}$ The carrier population at the AlN/GaN interface is shown in Fig. 6(b). Here, the sheet carrier density values 
are calculated assuming a 2DEG thickness of $2 \mathrm{~nm}$. As seen in Figs. 6(a), 6(b), and 2, the calculated sheet carrier density is in perfect agreement with the Hall measurements. Therefore we conclude that only a single subband is populated and that the origin of the beating effect is the spin splitting of the first energy subband.

To describe zero-field spin splitting, an effective magnetic field can be defined so that the spin Hamiltonian will be

$$
H=\frac{1}{2} \hbar \boldsymbol{\sigma} \cdot \mathbf{B}_{\mathrm{eff}}\left(\mathbf{k}_{\|}\right),
$$

where $\boldsymbol{\sigma}$ is the Pauli matrices vector and the $\mathbf{k}_{\|}$is the inplane wave vector. ${ }^{38}$ Magnitude and the direction of the effective magnetic field are depending on in-plane wave vector as seen on Eq. (2). For the single subband case, the in-plane wave vector dependent spin-splitting can be given by ${ }^{38}$

$$
\Delta\left(\mathbf{k}_{\|}\right) \hbar\left|\mathbf{B}_{\text {eff }}\right| .
$$

A spin-orbit Hamiltonian was proposed by Bychkov and Rashba to represent spin-orbit interactions as $H=\alpha(\boldsymbol{\sigma}$ $\times \mathbf{k}) \cdot \hat{\mathbf{z}}$. ${ }^{39}$ Here, $\alpha$ is the structure dependent parameter; spinorbit coupling constant and $\hat{\mathbf{z}}$ is the unit vector along the growth direction. So the effective magnetic field can be found as

$$
\mathbf{B}_{\text {so }}=(2 / \hbar) \alpha \mathbf{k} \times \hat{\mathbf{z}} \text {. }
$$

Effective magnetic field written in Eq. (4) concludes a spin-splitting value of $\Delta=2 \alpha k_{\|}$. Zero-field spin splitting gives rise to a similar beating pattern to that related to the magnetointersubband scattering (MIS) effect and, therefore, the exact calculation of the subband positions is also important for the investigation of the spin related beating pattern. ${ }^{40}$ The MIS effect occurs when a second subband is populated. ${ }^{41}$ In $\mathrm{AlGaN} / \mathrm{GaN}$ with a high $\mathrm{Al}$ composition or AlGaN/AlN/GaN structures, MIS becomes increasingly important compared to the zero-field spin splitting at elevated temperatures and when the intersubband energy separation is small. ${ }^{18}$ According to our calculations shown in Figs. 6(a) and 6(b), our samples have single subband occupancy with a high carrier density, in which the beating of the SdH oscillations is related to the zero-field spin splitting. The relation between the $\mathrm{SdH}$ frequencies and the carrier densities of spin-up (spin-down) electrons is ${ }^{18}$

$$
f_{\uparrow(\downarrow)}=\frac{h n_{\uparrow(\downarrow)}}{e},
$$

where $f_{\uparrow(\downarrow)}$ is the $\mathrm{SdH}$ frequency for spin-up (spin-down) electrons and its value is measured from the insert of Fig. 4 as 202.7 T and 226.1 T for spin-up and spin down electrons, respectively. Using Eq. (5), the carrier densities of spin-up and spin-down electrons are calculated as $n_{\uparrow}=5.46 \times 10^{12}$ and $n_{\downarrow}=4.90 \times 10^{12} \mathrm{~cm}^{-2}$. The total carrier density $\left(n_{\uparrow}+n_{\downarrow}\right)$ is consistent with the Hall sheet carrier density of 1.05 $\times 10^{13} \mathrm{~cm}^{-2}$ within an experimental accuracy of about $3 \%$.

Once the carrier densities of spin-up and spin-down electrons are known, the spin-orbit coupling parameter $\alpha$ can be found using the relation ${ }^{42}$

$$
\alpha=\frac{\Delta n \hbar^{2}}{m^{*}} \sqrt{\frac{\pi}{2\left(n_{s}-\Delta n\right)}} .
$$

Here, $\Delta n$ is the difference in carrier densities of spin-up and spin-down electrons $\Delta n=5.6 \times 10^{11} \mathrm{~cm}^{-2}$ and $n_{s}=n_{\uparrow}$ $+n_{\downarrow}$. From Eq. (6), we obtain the spin-orbit coupling parameter, $\alpha=7.85 \times 10^{-12} \mathrm{eV} \mathrm{m}$. While this value is much larger than that found in other heterostructure systems such as InAs/GaSb, $\quad \operatorname{In}_{x} \mathrm{Ga}_{1-x} \mathrm{As} / \mathrm{In}_{0.52} \mathrm{Al}_{0.48} \mathrm{As}, \quad \mathrm{In}_{0.53} \mathrm{Ga}_{0.47} \mathrm{As} /$ $\mathrm{In}_{0.77} \mathrm{Ga}_{0.23} \mathrm{As} / \mathrm{InP},{ }^{42-44}$ it agrees with the values of $\alpha$ for AlGaN/GaN heterostructures. ${ }^{15,38}$ In III-N structures, the higher value of the spin-orbit coupling parameter is likely to be caused by the strong internal electric fields induced by piezoelectric and spontaneous polarization. ${ }^{8,45}$ The calculated spin-orbit coupling parameter is a sum of the Rashba parameter $\alpha_{R}$ and a coupling parameter associated with the bulk inversion asymmetry in wurtzite quantum wells $\alpha_{\text {WBIA. }}{ }^{12}$ Zero-field spin splitting energy $(\Delta)$ can be calculated, therefore, from effective spin-orbit coupling parameter. The calculated value of $2 \alpha k$ is $12.75 \mathrm{meV}$. This is in good agreement with the spin-split energy of $12.30 \mathrm{meV}$ obtained from the $\mathrm{SdH}$ frequencies as

$$
E_{\uparrow}-E_{\downarrow}=\frac{2 \pi \hbar^{2} \Delta n}{m^{*}} .
$$

The list of the growth and electrical parameters are listed together with the spin related parameters and experimental methods used in the evaluation of the spin-splitting energy in $\mathrm{AlGaN} / \mathrm{GaN}$ and $\mathrm{AlGaN} / \mathrm{AlN} / \mathrm{GaN}$ obtained by us and others $^{10,12,15-21}$ is given in Table I.

Figure 7 shows the zero-field spin splitting energy versus sheet carrier density data from Table I. The WAL measurement results are shown with empty symbols and $\mathrm{SdH}$ measurements are shown with filled symbols. It is clear that there is a significant disagreement between WAL and $\mathrm{SdH}$ results. The WAL measurements show good agreement with the theoretical estimate based on the Rashba coupling mechanism. ${ }^{24}$ It should be noted that the $\mathrm{SdH}$ measurements fail in samples with sheet carrier densities below $n_{S} \sim 6 \times 10^{-12} \mathrm{~cm}^{-2}$ as a result of the lack of beating patterns. However, at sheet carrier densities above $6 \times 10^{-12} \mathrm{~cm}^{-2}$, the spin-splitting energy values begin from Rashba value and increase with the increasing sheet carrier density. This increase could be caused by the $\Delta_{C 1}-\Delta_{C 3}$ coupling, where the band folding generates two conduction bands, and by the lack of an inversion symmetry effect. ${ }^{25}$ An increase in the zero-field spin splitting with the increasing sheet carrier density is also expected according to the theoretical work by Litvinov. ${ }^{24}$ However, the reason for the disagreement between the SdH and WAL measurements is not clear to us.

\section{CONCLUSION}

We studied the magnetoresistivity of a 2DEG in AlGaN/ AlN/GaN/AlN heterostructures at low temperatures $(T$ $=2 \mathrm{~K}$ ) and high magnetic fields (up to $14 \mathrm{~T}$ ). We confirmed that the 2DEG has high mobility and high sheet carrier density and that the effect of any parallel conduction channel is negligible. We observed a beating effect in the $\mathrm{SdH}$ oscilla- 
TABLE I. Growth, electrical and spin related parameters, and experimental method used to calculate the spin-splitting energy for our AlGaN/AlN/GaN/AlN samples and for $\mathrm{AlGaN} / \mathrm{GaN}$ and $\mathrm{AlGaN} / \mathrm{AlN} / \mathrm{GaN}$ structures in the literature (Refs. 10, 12, and 15-21).

\begin{tabular}{|c|c|c|c|c|c|c|c|c|}
\hline Sample & $\begin{array}{c}T \\
(\mathrm{~K})\end{array}$ & $x(\mathrm{Al})$ & 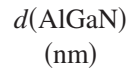 & $\begin{array}{l}d(\mathrm{AlN}) \\
(\mathrm{nm})\end{array}$ & $\begin{array}{c}n_{s} \\
\left(1 \times 10^{12} \mathrm{~cm}^{-2}\right)\end{array}$ & $\begin{array}{l}\text { Experimental } \\
\text { method }\end{array}$ & $\begin{array}{c}\alpha^{*} \\
\left(1 \times 10^{-12} \mathrm{eV} \mathrm{m}\right)\end{array}$ & $\begin{array}{c}2 \alpha^{*} k \\
(\mathrm{meV})^{\mathrm{a}}\end{array}$ \\
\hline Single sample type ${ }^{b}$ & 0.1 & 0.3 & 20 & 0 & 6.20 & WAL & 0.85 & 1.06 \\
\hline Sample $C^{\mathrm{c}}$ & 1.6 & 0.15 & 25 & 1 & 4.90 & WAL & 0.55 & 0.61 \\
\hline Sample $3^{\mathrm{d}}$ & 1.2 & 0.25 & $30^{\mathrm{e}}$ & 0 & 8.96 & $\mathrm{SdH}$ & 6.18 & 9.27 \\
\hline Sample $A^{\mathrm{f}}$ & 2 & 0.11 & 30 & 0 & 6.6 & $\mathrm{SdH}$ & 2.4 & 3.09 \\
\hline Sample $B^{f}$ & 2 & 0.25 & 28 & 0 & 8.5 & $\mathrm{SdH}$ & 2.8 & 4.09 \\
\hline Sample $C^{\mathrm{f}}$ & 2 & 0.28 & 25 & 0 & 12 & $\mathrm{SdH}$ & 4.5 & 7.81 \\
\hline Single sample type $\mathrm{e}^{\mathrm{g}}$ & 1.5 & 0.11 & 23 & 0 & 5.5 & $\mathrm{SdH}$ & 0.94 & 1.11 \\
\hline Single sample type ${ }^{\mathrm{h}}$ & 2 & 0.11 & 30 & 0 & 7.00 & $\mathrm{SdH}$ & 2.20 & 2.92 \\
\hline Sample B ${ }^{\mathrm{i}}$ & 0.3 & 0.12 & 16 & 0 & 1.8 & WAL & 0.6 & 0.40 \\
\hline Sample $1^{\mathrm{j}}$ & 1 & 0.15 & 35 & 0 & 4.21 & WAL & 0.85 & 0.87 \\
\hline Sample $A^{k}$ & 1.8 & 0.1 & 25 & 1 & 0.8 & WAL & 5.0 & 0.22 \\
\hline Sample $\mathrm{B}^{\mathrm{k}}$ & 1.8 & 0.15 & 25 & 1 & 1.7 & WAL & 5.0 & 0.33 \\
\hline Sample $C^{k}$ & 1.8 & 0.25 & 25 & 1 & 3.1 & WAL & 5.0 & 0.44 \\
\hline Sample $\mathrm{D}^{\mathrm{k}}$ & 1.8 & 0.3 & 25 & 1 & 5.3 & WAL & 5.0 & 0.58 \\
\hline Sample $E^{\mathrm{k}}$ & 1.8 & 0.35 & 25 & 1 & 8.1 & WAL & 5.0 & 0.71 \\
\hline This study & 2 & 0.22 & 25 & 1.5 & 10.50 & $\mathrm{SdH}$ & 7.85 & 12.75 \\
\hline
\end{tabular}

${ }^{\mathrm{a}}$ Calculated using Hall carrier densities for the wave vector.

${ }^{\mathrm{b}}$ Reference 10.

${ }^{c}$ Reference 12.

${ }^{\mathrm{d}}$ Reference 15.

${ }^{e} 20 \mathrm{~nm} n$-type with $1.7 \times 10^{17} \mathrm{~cm}^{-3} \mathrm{Si}$ doping $+10 \mathrm{~nm}$ undoped.

${ }^{\mathrm{f}}$ Reference 16.

${ }^{\mathrm{g}}$ Reference 17.

${ }^{\text {h }}$ Reference 18.

${ }^{\mathrm{i}}$ Reference 19.

${ }^{\mathrm{j}}$ Reference 20.

${ }^{\mathrm{k}}$ Reference 21.

tions, which was attributed to zero-field spin splitting. This conclusion was supported with a one-dimensional selfconsistent nonlinear Schrödinger-Poisson equation solution and analytic calculations of spin carrier density. For the zerofield spin splitting mechanism, an effective spin-orbit coupling parameter along with zero-field spin splitting energy values were calculated and compared with the literature. To

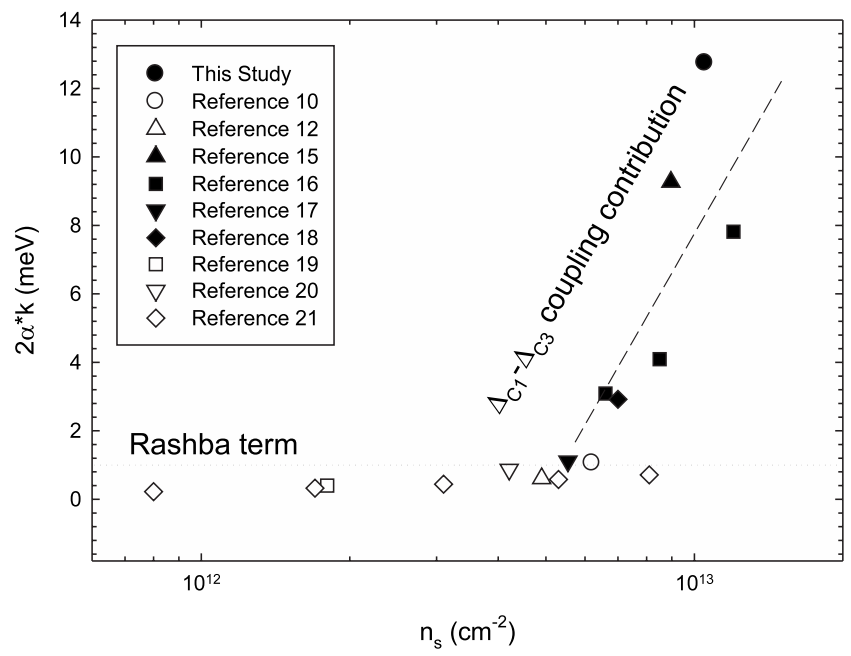

FIG. 7. Sheet carrier density dependent zero-field spin splitting energy values using data listed in Table I. Filled and empty symbols represent SdH and WAL experiment results, respectively. Dashed line is guide to the eyes and dotted line represents the theoretical value based on the Rashba coupling mechanism. our knowledge, the zero-field spin splitting energy observed in our structure $(12.75 \mathrm{meV})$ is the largest value reported so far in the literature for the GaN-based systems. Comparison with the recent literature indicates disagreement between the values obtained using WAL and $\mathrm{SdH}$ experiments. SdH measurements cannot be used for sheet carrier densities under $6 \times 10^{-12} \mathrm{~cm}^{-2}$ due to lack of beating patterns where the WAL measurements show good agreement with the theoretical values based on the Rashba coupling mechanism. ${ }^{24}$ In $\mathrm{SdH}$ experiments at sheet carrier densities above 6 $\times 10^{-12} \mathrm{~cm}^{-2}$, the spin-splitting energy values begin from Rashba value and increase with the increasing sheet carrier density. This increase could be caused by the $\Delta_{C 1}-\Delta_{C 3}$ coupling. It is also predicted by the theoretical work by Litvinov. $^{24}$

\section{ACKNOWLEDGMENTS}

This work is supported by the State of Planning Organization of Turkey under Grant No. 2001K120590 and by TUBITAK under Project Nos. 104E090, 105E066, and 105A005. S. B. Lisesivdin acknowledges a 2214 coded international research scholarship from TUBITAK (Turkey), A.P. acknowledges support from the Engineering and Physical Sciences Research Council (UK) and E.O. acknowledges partial support from the Turkish Academy of Sciences (Turkey).

${ }^{1}$ M. Asif Khan, J. N. Kuznia, J. M. Van Hove, N. Pan, and J. Carter, Appl. 
Phys. Lett. 60, 3027 (1992).

${ }^{2}$ Z. Fan, C. Lu, A. E. Botchkarev, H. Tang, A. Salvador, O. Aktas, W. Kim, and H. Morkoc, Electron. Lett. 33, 814 (1997).

${ }^{3}$ U. K. Mishra, Y.-F. Wu, B. P. Keller, S. Keller, and S. P. DenBaars, IEEE Trans. Microwave Theory Tech. 46, 756 (1998).

${ }^{4}$ N. Maeda, T. Saitoh, K. Tsubaki, T. Nishida, and N. Kobayashi, Jpn. J. Appl. Phys., Part 2 38, L799 (1999).

${ }^{5}$ W. Knap, H. Alause, J. M. Bluet, J. Camassel, J. Young, M. Asif Khan, Q. Chen, S. Huant, and M. Shur, Solid State Commun. 99, 195 (1996).

${ }^{6}$ W. Knap, S. Contreras, H. Alause, C. Skierbiszewski, J. Camassel, M. Dyakonov, J. L. Robert, J. Yang, Q. Chen, M. Asif Khan, M. L. Sadowski, and S. Huant, Appl. Phys. Lett. 70, 2123 (1997).

${ }^{7}$ O. Ambacher, B. Foutz, J. Smart, J. R. Shealy, N. G. Weimann, K. Chu, M. Murphy, A. J. Sierakowski, W. J. Schaff, L. F. Eastman, R. Dimitrov, A. Mitchell, and M. Stutzmann, J. Appl. Phys. 87, 334 (2000).

${ }^{8}$ F. Bernardini, V. Fiorentini, and D. Vanderbilt, Phys. Rev. B 56, R10024 (1997).

${ }^{9}$ I. Zutic, J. Fabian, and S. Das Sarma, Rev. Mod. Phys. 76, 323 (2004).

${ }^{10}$ N. Thillosen, Th. Schäpers, N. Kaluza, H. Hardtdegen, and V. A. Guzenko, Appl. Phys. Lett. 88, 022111 (2006).

${ }^{11}$ S. A. Wolf, D. D. Awschalom, R. A. Buhrman, J. M. Daughton, S. von Molnár, M. L. Roukes, A. Y. Chtchelkanova, and D. M. Treger, Science 294, 1488 (2001).

${ }^{12}$ C. Kurdak, N. Biyikli, U. Ozgur, H. Morkoc, and V. I. Litvinov, Phys. Rev. B 74, 113308 (2006).

${ }^{13}$ P. D. Dresselhaus, C. M. A. Papavassiliou, R. G. Wheeler, and R. N. Sacks, Phys. Rev. Lett. 68, 106 (1992).

${ }^{14}$ K. Tsubaki, N. Maeda, T. Saitoh, and N. Kobayashi, Appl. Phys. Lett. 80, 3126 (2002).

${ }^{15}$ I. Lo, J. K. Tsai, W. J. Yao, P. C. Ho, L.-W. Tu, T. C. Chang, S. Elhamri, W. C. Mitchel, K. Y. Hsieh, J. H. Huang, H. L. Huang, and W.-C. Tsai, Phys. Rev. B 65, 161306 (2002).

${ }^{16}$ N. Tang, B. Shen, K. Han, F.-C. Lu, F.-J. Xu, Z.-X. Qin, and G.-Y. Zhang, Appl. Phys. Lett. 93, 172113 (2008).

${ }^{17}$ W. Z. Zhou, T. Lin, L. Y. Shang, L. Sun, K. H. Gao, Y. M. Zhou, G. Yu, N. Tang, K. Han, B. Shen, S. L. Guo, Y. S. Gui, and J. H. Chu, J. Appl. Phys. 104, 053703 (2008).

${ }^{18}$ N. Tang, B. Shen, M. J. Wang, K. Han, Z. J. Yang, K. Xu, G. Y. Zhang, T. Lin, B. Zhu, W. Z. Zhou, and J. H. Chu, Appl. Phys. Lett. 88, 172112 (2006).

${ }^{19}$ S. Schmult, M. J. Manfra, A. Punnoose, A. M. Sergent, K. W. Baldwin, and R. J. Molnar, Phys. Rev. B 74, 033302 (2006).

${ }^{20}$ N. Thillosen, S. Cabanas, N. Kaluza, V. A. Guzenko, H. Hardtdegen, and Th. Schäpers, Phys. Rev. B 75, 241311(R) (2006).

${ }^{21}$ H. Cheng, N. Biyikli, U. Ozgur, C. Kurdak, H. Morkoc, and V. I. Litvinov, Physica E 40, 1586 (2008).

${ }^{22}$ E. B. Olshanetsky, Z. D. Kvon, S. Sassine, J. C. Portal, H. I. Cho, and J.
H. Lee, Appl. Phys. Lett. 92, 242112 (2008).

${ }^{23}$ W. Weber, S. D. Ganichev, S. N. Danilov, D. Weiss, W. Prettl, Z. D. Kvon, V. V. Belkov, L. E. Golub, H.-I. Cho, and J.-H. Lee, Appl. Phys. Lett. 87, 262106 (2005).

${ }^{24}$ V. I. Litvinov, Phys. Rev. B 68, 155314 (2003).

${ }^{25}$ I. Lo, W. T. Wang, M. H. Gau, S. F. Tsay, and J. C. Chiang, Phys. Rev. B 72, 245329 (2005).

${ }^{26}$ A. F. Brana, C. Diaz-Paniagua, F. Batallan, J. A. Garrido, E. Munoz, and F. Omnes, J. Appl. Phys. 88, 932 (2000).

${ }^{27}$ D. G. Seiler and A. E. Stephens, in Landau Level Spectroscopy, edited by G. Landwehr and E. I. Rashba (North-Holland, Amsterdam, 1991), Vol. 2, p. 1031.

${ }^{28}$ D. R. Leadley, R. J. Nicholas, J. J. Harris, and C. T. Foxon, Semicond. Sci. Technol. 4, 885 (1989).

${ }^{29}$ H. Celik, M. Cankurtaran, A. Bayrakli, E. Tiras, and N. Balkan, Semicond. Sci. Technol. 12, 389 (1997).

${ }^{30}$ E. Tiras, M. Cankurtaran, H. Celik, A. B. Thoms, and N. Balkan, Superlattices Microstruct. 29, 147 (2001).

${ }^{31}$ S. Acar, S. B. Lisesivdin, M. Kasap, S. Ozcelik, and E. Ozbay, Thin Solid Films 516, 2041 (2008).

${ }^{32}$ S. B. Lisesivdin, A. Yildiz, S. Acar, M. Kasap, S. Ozcelik, and E. Ozbay, Appl. Phys. Lett. 91, 102113 (2007).

${ }^{33}$ S. B. Lisesivdin, S. Acar, M. Kasap, S. Ozcelik, S. Gokden, and E. Ozbay, Semicond. Sci. Technol. 22, 543 (2007).

${ }^{34}$ S. B. Lisesivdin, A. Yildiz, S. Acar, M. Kasap, S. Ozcelik, and E. Ozbay, Physica B 399, 132 (2007).

${ }^{35}$ I. Lo, W. C. Mitchel, M. Ahoujja, J.-P. Cheng, A. Fathimulla, and H. Mier, Appl. Phys. Lett. 66, 754 (1995).

${ }^{36}$ S. Birner, S. Hackenbuchner, M. Sabathil, G. Zandler, J. A. Majewski, T. Andlauer, T. Zibold, R. Morschl, A. Trellakis, and P. Vogl, Acta Phys. Pol. A 110, 111 (2006).

${ }^{37}$ J. A. Garrido, J. L. Sanchez-Rojas, A. Jimenez, E. Munoz, F. Omnes, and P. Gibart, Appl. Phys. Lett. 75, 2407 (1999).

${ }^{38}$ E. A. de Andrada e Silva, Phys. Rev. B 46, 1921 (1992).

${ }^{39}$ Y. A. Bychkov and E. I. Rashba, J. Phys. C 17, 6039 (1984).

${ }^{40}$ N. Tang, B. Shen, Z. W. Zheng, J. Liu, D. J. Chen, J. Lu, R. Zhang, Y. Shi, Y. D. Zheng, Y. S. Gui, C. P. Jiang, Z. J. Qiu, S. L. Guo, J. H. Chu, K. Hoshino, T. Someya, and Y. Arakawa, J. Appl. Phys. 94, 5420 (2003).

${ }^{41}$ T. H. Sander, S. N. Holmes, J. J. Harris, D. K. Maude, and J. C. Portal, Phys. Rev. B 58, 13856 (1998).

${ }^{42}$ Th. Schäpers, G. Engels, J. Lange, T. Klocke, M. Hollfelder, and H. Lüth, J. Appl. Phys. 83, 4324 (1998).

${ }^{43}$ J. Luo, H. Munekata, F. F. Fang, and P. J. Stiles, Phys. Rev. B 41, 7685 (1990).

${ }^{44}$ B. Das, S. Datta, and R. Reifenberger, Phys. Rev. B 41, 8278 (1990).

${ }^{45}$ K. S. Cho, T.-Y. Huang, H.-S. Wang, M.-G. Lin, T.-M. Chen, C.-T. Liang, and Y. F. Chen, Appl. Phys. Lett. 86, 222102 (2005). 